\title{
ALGUNAS CONSIDERACIONES SOBRE 35 CASOS DE NEUMOTORAX ESPONTANEO EN EL NINO
}

\author{
Por los Dres. J. BAUZA, E. ROSENBLUT y C ALLIENDE. \\ Hospital Roberto del Rio. Servicio del Prof. Seroggie.
}

Fué Itar, quien en 1803 llamó neumotórax a la entrada brusca de aire en la cavidad pleural.

Se distinguen 3 clases de neumotórax:

1. Neumotórax espontáneo.

2. Neumotórax traumático.

3. Neumotórax terapéutico o artificial.

Su enumeración nos ahorra la definición de cada uno de ellos. En esta presentación nos ocuparemos en forma exclusiva del neumotórax espontáneo.

Las circunstancias y condiciones previas en que aparece este tipo de neumotórax son múltiples y de ahí que se hallan adoptado diversas clasificaciones.

Coope distingue:

1. Neumotórax benigno, cicatricial o buloso:

2. Neumotórax que complica a un enfisema antiguo.

3. Neumotórax que complica una tuberculosis.

4. Neumotórax que obedece a otras causas.

Esta clasificación no deja bien establecidas las diferencias etiológicas fundamentales, por lo que hemos adoptado la siguiente:

1. Neumotórax en apariencia primitivo 0 esencial.

2. Neumotórax secundario a bronconeumopatias.
El neumotórax en apariencia primitivo se presenta en sujetos hasta entonces en buenas condiciones aparentes de salud, sin que nada permita preverlo, sin que los exámenes clínico y radiológico, después de este accidente, permita reconocer lesiones respiratorias. Las circunstancias en que puede aparecer son múltiples, desde un esfuerzo pequeño (caminar, llorar, etc.) o aun durante el sueño, $\sin$ que existan antecedentes de ninguna actividad intensa.

Las formas secundarias pueden ser consecutivas a enfermedades respiratorias de la más diversa índole:

a) Agudas: como bronquitis aguda, bronconeumonía y neumonía.

b) Crónicas: como bronqulectasia, abscesos pulmonares, enfisema de los viejos, neoplasias pulmonares, micosis, tuberculosis pulmonar activa o eicatricial.

Guillard, en 1883, fué el primero en señalar que el neumotórax espontáneo es debido a la rotura de una vesícula de enfisema sub pleural, nombre dado en 1933 por los autores argentinos Pardal y Mazzei. Esta opinión es compartida por la mayoría de los autores que se han preocupado del problema.

El mecanismo de producción del enfisema sub pleural es difícil de explicar en los casos que denominamos neumotórax primitivo y que corresponde a la forma cicatricial, benigna 0 bulosa de la clasificación de Coope. 
Siempre se supone una alteración local previa que no es reconocible en absoluto; se trata, por lo tanto, de lesiones mínimas que pueden tener diversa etiología.

El esfuerzo como causa única, sin la existencia de alteraciones locales previas, se rechaza como origen de la afección. La experimentación demuestra que durante el esfuerzo la presión alveolar permanece invariable, sólo aumenta cuando, por diversas circunstancias, se reduce o limita la amplitud de la caja toráxica. La práctica diaria nos muestra que siendo muy frecuentes los esfuerzos violentos y prolongados (parto), son poco numerosos los casos de neumotórax espontáneo debidos a esta causa. Tampoco debemos olvidar que hay neumotórax espontáneos que se presentan durante el reposo y el sueño.

Las alteraciones locales están constituídas por lesiones cicatriciales no detectables ni aún a la radiografía dirigida.

En el niño menor los procesos inflamatorios agudos son los principales causantes que al actuar sobre el bronquio y debilitar la pared alveolar (con la consecuente pérdida de elasticidad y de las propiedades específicas de expansión $\mathrm{y}$ retracción) van a favorecer la ruptura. Además de la infección, se indican como causas algunas alteraciones locales ligadas al crecimiento: Miller y Engel han llamado la atención que durante el crecimiento se producen vesículas puimonares nuevas; por lo tanto, detenciones localizadas de este crecimiento cambian las condiciones normales. Engel textualmente dice: "La frecuencia de enfisema en los nin̄os se debe, en su mayor parte, a la falta de desarrollo del armazón del pulmón, siendo la elasticidad del mismo menor que en el adulto; la hiperdistensión agota fácilmente el poder elástico del tejido, con lo que resulta una expansión persistente". Y más adelante, refiriéndose al recién nacido, "el pulmón del recién nacido es cuasi enfisematoso, se encuentran más conductilios que alvéolos".
Igualmente, pueden invocarse deformaciones anatómicas en el sentido de malformaciones congénitas, de origen constitucional o hereditario, malformaciones vasculares, etc.

Estas alteraciones locales de uno u otro origen, hacen que se produzca una debilidad de la pared alveolar; por pérdida de la elasticidad y aumento de la expandibilidad en un momento dado, rotura de ella, con desgarro de la limitante elástica profunda de la pleura visce-

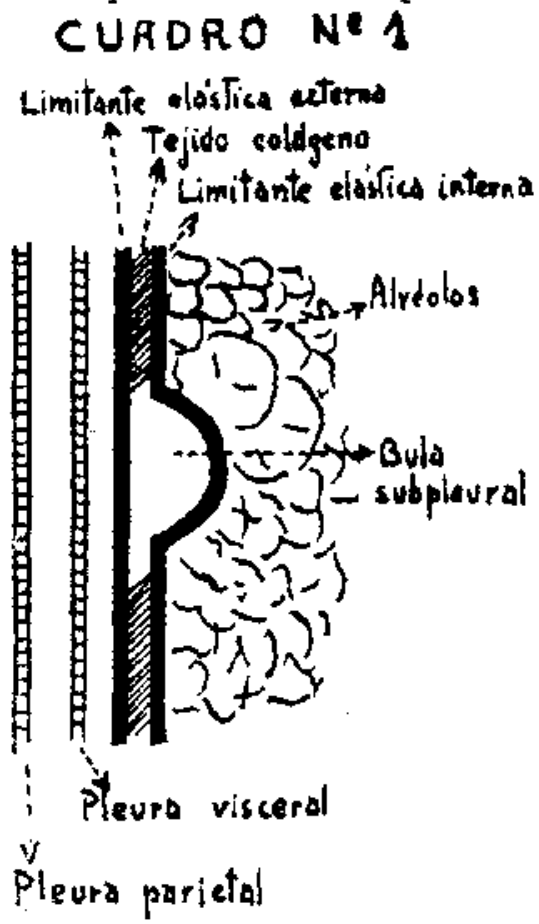

ral, dando lugar a la formación de una cámara de aire entre las dos limitantes elásticas que forman la serosa visceral, sin participación de la capa superficial recubierta de epitelio. Entre las dos limitantes elásticas hay tejido colágeno, por el cual van los vasos, venas y linfáticos.

La comunicación de esta cámara por una efracción de la pleura visceral con la cavidad pleural es la causa del neumotórax espontáneo. La producción y existencia de las vesículas sub-pleurales son condición "sine-qua-non" en la gé nesis de este tipo de neumotórax.

Ia "bulla" (nombre dado por Miller) es una ampolla de enfisema producida 
por la destrucción y rotura de los tabiques interalveolares con formación, a su vez, de una cámara de aire sub-pleural, que va a desalojar en su totalidad las capas que constituyen la pleura visceral. Presupone siempre estenosis bronquial, y como se comprende, la producción de un neumotórax es más difícil, ya que mientras el enfisema es intersticial pleural, la bulla ocupa los intersticios alveolares, es sub-pleural y rechaza a la pleura.

Las vesículas pueden ser únicas o múltiples, pequeñas o gígantes, confundiéndose muchas veces con formaciones quísticas pulmonares.

Para Sargent y parte de la escuela francesa, las cosas pasarían de otra manera: el neumotórax se produciría por la rotura de una cicatriz tuberculosa. Etiologia difícil de concordar cuando la tuberculosis no existe.

No nos ocuparemos del neumotórax como complicación del enfisema pulmonar crónico del adulto y principalmente del viejo, para dedicarnos a aquellas formas de neumotórax secundario, más frecuentes en pediatría.

De las formas secundarias nos referiremos, en primer lugar, a aquellos neumotórax que complican la tuberculosis del niño en cualquiera de sus formas o períodos.

E1 neumotórax de la primo infección no complicada es benigno en su evolución la mayoría de las veces; en cambio, el neumotórax, que complica la tuberculosis caseosa y úlcero-caseosa, aun más cuando es bilateral, es muy grave, ya que la capacidad vital y funcional está muy afectada.

La complicación pleural recorrería en esta última eventualidad 3 etapas:

1) Inicial, que va acompañado la mayoría de las veces de schock pleural. Según el tipo $\mathrm{y}$ extensión de la lesión $\mathrm{y}$ grado de compromiso del otro pulmón, se puede producir la muerte, o pasar al segundo período.

2) Neumotórax estabilizado, rara vez es éste el estado final, volviendo las cosas a la situación inicial, por reabsorción del aire y cierre de la rotura pleu- ral. La mayoría de los casos pasan al tercer período.

3) Neumotórax con derrame que puede ser sero-fibrinoso y con más frecuencia purulento. La evolución de este segundo caso puede ser maligna y rápida por infección secundaria del líquido pleural. En algunos casos estas pleuresías se hacen crónicas y en general son de mal pronóstico. Otras veces el derra. me sero-fibrinoso se va transformando muy lentamente en derrame purulento.

La iniciación y producción del neumotórax puede ser repentino, gradual o insidioso.

Según su extensión pueden ser totales o parciales.

Según el grado de colapso, completos o incompletos.

Finalmente, según el grado de comunicación con el exterior, puede ser cerrado, abierto, a tensión o a válvula.

1) Neumotórax cerrado: Se produce por retracción sobre sí mismo de los bordes del desgarro. Se puede agregar escaso derrame producido por irritación pieural. El aire se absorbe lentamente y el pulmón se reexpande. Inicialmente puede haber en este tipo presiones positivas. Se caracteriza por su benignidad, falta de signos de infección, temperatura y otros; algunas veces no presenta lesiones previas. (Cuadro $\mathrm{N}^{\circ} 2$ ).

2) Neumotórax abierto: $\mathrm{El}$ borde superior o inferior del desgarro, más frecuentemente el superior, es mantenido sujeto a la pleura parietal con retracción del otro borde. Hay libre pasaje de aire a través de la fístula bronquial, con lo que la presión pleural se igualó a la atmostérica. (Cuadro $\mathrm{N}^{0} 3$ ).

3) Neumotórax a tensión o válvula: El pulmón colapsado, bajo ciertas condiciones que signifiquen esfuerzo efectuado por la glotis cerrada (como la tos), da paso a través de la fístula a la entrada de aire, el que no puede salir en la expiración. Mientras más aire pasa, mayor valor positivo toma la presión pleural. 
Se comprende la importancia del neumotórax a válvula por su acción sobre la mecánica cardio-respiratoria; desviación del mediastino, compresión de los grandes vasos, etc. Esto explica el síndrome cardiaco, que muchas veces acompaña a este cuadro (neumotorax sofocante).

CUADRO NO 2

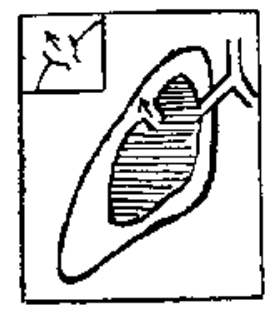

N.E. debide a ruptura de una burbuja subtefeural

$$
\text { CUADRO NE } 3
$$

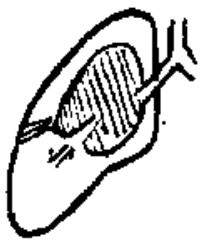

C ME. aburto. Lo

pistula se man.

tiene obierta por

aherencia pleural

En algunos casos la patogenia del neumotórax sofocante es distinta. Para Macklin (en 1939) y Hamman (en 1945), por medio de un mecanismo valvular (estenosis bronquial) se produciría la ruptura del alvéolo hacia el espacio perivascular con irrupción de aire, el que siguiendo el trayecto de los grandes vasos iría a producir el enfisema mediastinal a hiper-presión; este aire seguiría dos caminos: ya sea hacia el cuello, produciendo el enfisema subcutáneo, y hacia la pleura mediastínica, cuya rotura ocasiona el neumotórax.

Uno de nuestros enfermos con una tuberculosis úlcero-caseosa bilateral presentó bruscamente enfisema del cuello; al día siguiente presenta el cuadro típico de schock pleural, acompañado de los signos de neumotórax espontáneo con muerte del enfermo, encontrándose en la anatomía patológica ruptura de la pleura mediastínica.

Montalbán, en 1947, describe 5 casos de neumomediaștino en el recién nacido, en que el mecanismo habría sido el descrito,

Además de la sintomatología clínica podemos diferenciar las tres formas anteriormente descritas, tomando la presión con un aparato de neumotórax; en el neumotórax abierto la presión es cerca de 0 . con pequeñas oscilaciones, menos 2 en inspiración y más 2 en la expiración. El neumotórax cerrado dará presiones negativas con menos 10 menos 4 al final de la expiración. En cambio, el neumotórax a tensión da variaciones en mediciones sucesivas; menos $12 \quad 0$ más 8, o más 4 o más 18, pero sicmpre positivas.

La medición de las presiones es indispensable para indicación del tratamiento.

La introducción de aire o extracción de él no hacen variar las presiones en el neumotórax abierto.

En el cerrado, la introducción aumenta la presión y la mantiene alta por un tiempo, y la extracción es muy peligrosa, porque puede producirse un desgarro, cuyo resultado sería la formación de un neumotórax abierto.

En el neumotórax a tensión la introđưcción de aire es también peligrosa, porque puede producirse un neumotórax sofocante; $y$ el extraerlo en grandes cantidades pone en juego el mecanismo de válvula.

Síntomas: Se pueden dividir en signos locales y generales, siendo los locales los más importantes.

Signos generales: Tos, algunas veces por quintas y abscesos; dolor exagerado por Ios movimientos de la tos.

La brusquedad de aparición determina que los síntomas generales sean más alarmantes, con sensación de angustiadisnea $y$ dolor intenso, correspondiendo al llamado schock pleural. No hay que olvidar que muchas veces esta sintomatología revela una insuficiencia cardíaca aguda producida por el mismo neu- 
motórax. La disnea es un signo que muchas veces falta y cuando existe puede corresponder o ser síntoma de una insuficiencia pulmonar.

Estos sintomas son llamados de iniciación; están en relación con la cantidad de gas acumulado, existencia de adherencias, estado del pulmón opuesto y brusquedad de aparición. Algunas veces son tan atenuados que el neumotórax es sólo un hallazgo de radiología. Otros síntomas generales son palidez, temperatura en relación con la enfermedad desencadenante, tiraje, etc.

Los signos locales, juntos con los síntomas radiológicos, son los elementos más importantes en el diagnóstico del neumotórax espontáneo.

Entre ellos tenemos: disminución de la movilidad e incursión respiratoria. les.

Disminución de las vibraciones voca-

Timpanismo, aparición de sonoridad en zonas que antes estaban mate.

Desaparición de la macidez hepática y cardíaca.

Disminución o abolición del murmullo vesicular (silencio respiratorio).

Cuando el neumotórax está a alguna tensión, tenemos abombamiento del hemitórax correspondiente, resonancia anfórica o sub-macidez a la percusión. Soplo anfórico, timbre metálíco de los ruidos agregados, sucusión hipocrática, ruido de glu glu (estos últimos revelan presencia de líquidos).

En un caso que existía un neumotórax total sin derrame, éste evolucionó a hidroneumotórax en un plazo de $\mathbf{2 5}$ días (comprobación radiológica). Un neumotórax parcial que se puncionó se transformó en un pioneumotórax.

En los neumotórax parciales es sabido el rol que juegan las adherencias, las que tabicando la cavidad pleural pulmonar evitan que el neumotorax se haga total.

El estudio radiológico es fundamental para observar la retracción y desplazamiento del mediastino durante los movimientos respiratorios.

Diagnóstico: Cuando se trata del cuadro clásico, acompañado de los signos que hemos enunciado, el diagnóstico es fácil.

Se presenta dificultad cuando hay pobreza de signos y cuando éstos son comunes a otras enfermedades.

1) Empiemas o pleuresías sero-fibrinosas tuberculosas. Muchas veces es la radiología la medición de las presiones, entre otros exámenes, los que aclaran el cuadro.

2) En una tuberculosis, la aparición brusca de una granulia puede semejar el schock pleural, pero los signos clínicos y radiológicos son muy diferentes.

3) Cavernas gigantes: El diagnóstico es más difícil, ya que los signos clínicos son difíciles de precisar, y aun el diagnóstico es difícil para el radiólogo, especialmente en las cavernas altas.

4) Abscesos pulmonares: En algunas acasiones pueden presentarse ambas lesiones juntas (sepsis). Sólo la radiología y la correcta interpretación de los signos dará el diagnóstico.

5) Quiste hidatidico abierto: Servirá la radiografía y la broncografía, especialmente en los casos difíciles, ya que el medio de contraste va a caer en la cavidad.

6) Quistes aéreos gigantes y enfisemas bulosos: La forma redondeada, las variaciones de tamaño que sufre por la comunicación con el exterior en los movimientos respiratorios, son elementos que ayudan al diagnóstico.

Para facilidad de la exposición hemos refundido los quistes aéreos con el enfisema buloso, ya que siempre en este último hay comunicación con el exterior, aunque sea microscópico.

Tratamiento: En los neumotórax no complicados el tratamiento es reposo en cama y observación del enfermo; pulso, temperatura, respiración, calmando la tos. En los neumotórax sofocantes no debemos olvida $\vec{r}$ que van involucrados las insuficienclas respiratoria y cardíaca aguda, por lo cual la oxigenoterapia, los analépticos, el reposo riguroso, los analgésicos para calmar el dolor y las punciones evacuadoras cerradas para evi- 
ar la infección secundaria, son armas poderosas.

Es la disnea, cianosis y rechazo del mediastino lo que da la indicación para las punciones repetidas y pequeñas efectuadas con aparatos de neumotórax. Si ssto no basta, se podrá dejar el trócar zvacuador a permanencia fijado con tela adhesiva; otras veces se deja sondaje a permanencia con un tubo de goma, suyo extremo terminal se introduce en un recipiente con agua, mecanismo que permite la salida del aire y no su entrada. Debe insistirse en las precauciones para evitar la infección secundaria.

En un grado más avanzado se podría ir a la toracotomía amplia. Así mismo en los neumotórax que se mantienen largo tiempo, deberá intentarse su curación mediante medios quirúrgicos adecuados.

No hay que olvidar el tratamiento de la enfermedad desencadenante del neumotórax; si se trata de un proceso agudo se usarán los antibióticos correspondientes.

Es indudable que el uso adecuado de los antibióticos hará que disminuya el neumotórax como complicación de los cuadros agudos.

En los neumotórax complicados con empiema se recurrirá a la combinación de punciones evacuadoras y empleo de antibióticos.

En resumen, en el neumotórax sólo se hará un tratamiento especial cuando se encuentra a presión o cuando es persistente; en los casos restantes basta el tratamiento de la enfermedad causal o del empiema.

La elección del procedimiento quirúzgico a emplear depende de la lesión anatómica responsable.

Siguiendo la opinión de Etienne Bernard, en el caso de simples vesículas enfisematosas, previa pleuroscopía, se resecarán las adherencias y se tratará de provocar una sinfisis pleural por pulverización de talco. Las vesículas voluminosas o quistes aéreos, simples o en pequeño número, pueden ser tratadas por escisión, previa toracotomía.
Las lesiones enfisematosas o quísticas, localizadas en parte limitada del pulmón, requieren la lobectomía. Finalmente, en algunos casos de neumotórax crónico con engrosamiento pleural marcado, recurre a la decorticación, seguida en ocasiones de toracoplastía, para evitar la extrema expansión del lóbulo restante.

Es indudable que el neumotórax espontáneo tuberculoso requiere consideraciones especiales. Cuando se trate de un neumotórax que compiica una primo infección de evolución benigna, el tratamiento será la observación del enfermo y medidas de orden general. Cambiará el procedimiento a seguir cuando el neumotórax complica a una tuberculosis excavada uni o bilateral. Es aquí donde entran en juego las consideraciones sobre cada caso en particular, tomándose, en algunos, una conducta expectante e intervencionista en otros, a pesar de la gravedad que dicha terapéutica involucra. Los métodos quirúrgicos varían desde los menos inocuos hasta la neumectomía.

No es necesario insistir en la protección de los antibióticos correspondientes.

De ia bibliografía nacional anotamos que el Prof. Ariztía presentó 8 casos de neumotórax espontáneo en 1938; M. Neira, 8 casos en 1942; P. Oyarce, en 1946 presenta un caso en un niño de 3 meses y en 1949 los Dres. Matte y Pulido presentan 8 casos.

En esta oportunidad analizamos $\mathbf{3 5}$ casos recogidos en el Hospital Roberto del Río en un período de 12 años, estando incluídos entre ellos los 8 casos del Dr. Neira y el caso de Oyarce.

La edad de nuestros pacientes es la siguiente:

\section{Cuadro N: 4}

Edad $\quad \mathbf{N}^{\mathrm{n}}$ de casos

\begin{tabular}{lrl}
\hline $0-2$ años & 14 & $40 \%$ \\
$2-4$ años & 12 & $34.25 \%\{74.25 \%$ \\
$4-12$ años & 9 & $25.75 \%$ \\
\hline Total & 35 & $74.25 \%(0-4)$ años
\end{tabular}


Como vemos, el $74.2 \%$, o sea, aproximadamente las $3 / 4$ partes lo constituyen niños menores de 4 años. Esta mayor frecuencia se explica, además de la mayor morbilidad y presencia de cuadros respiratorios agudos, por la falta de maduración del estado inmunológico en la infancia, debido a la mayor fragilidad y labilidad de las estructuras pulmonares del niño menor; este predominio en el niño menor lo encontramos también en la presentación de Ariztía: en $\mathbf{5}$ casos son niños menores de 5 años.

Igualmente esta mayor fragilidad del niño menor es evidente en la presentación de Meneghello y Hasbun, de cuyos 13 casos, 2 solamente son mayores de 4 años.

Etiología: Entre los casos que enumeramos no contamos con las formas que hemos llamado esenciales; todos son secundarios a otras afecciones respiratorias en la forma que señala el cuadro No 5 .

\section{Cuadro $N^{*} 5$}

\section{Etiologia.}

A.-Neum. consecutivos a procesos agudos.

\begin{tabular}{ccccc} 
& $\begin{array}{c}-2 \\
\text { añas }\end{array}$ & -4 & +4 & Total \\
aros & años & \\
$\begin{array}{c}\text { nempuitis, bronc., } \\
\text { nemsis y abscesos } \\
\text { pulmonares }\end{array}$ & 12 & 9 & 2 & 23 \\
\hline
\end{tabular}

B.-Neum, consecutivos a tuberculosis.

\begin{tabular}{|c|c|c|c|c|}
\hline & $\frac{2}{\text { años }}$ & $\frac{-4}{\text { años }}$ & $\frac{+4}{\text { años }}$ & Total \\
\hline $\begin{array}{l}\text { Primaria no exca- } \\
\text { vada. }\end{array}$ & -2 & 1 & 1 & 4 \\
\hline $\begin{array}{l}\text { Primarla excavada } \\
\text { Post primaria excav. } \\
\text { (úlcero caseosa) }\end{array}$ & 1 & 0 & 1 & 2 \\
\hline
\end{tabular}

Aunque el número de enfermos no permite sacar conclusiones estadísticas, vemos también que en el menor de 4 años las afecciones respiratorias agudas forman la gran mayoría $(84.6 \%)$, mientras las formas crónicas el $45.4 \%$, lo que es explicable, como ya lo hemos dicho, por la mayor frecuencia de las afecciones catarrales agudas, sepsis, etc. en el niño menor y por la menor incidencia de la afección tuberculosa a estas edades. A la inversa en el niño mayor de 4 años, existe un discreto predominio de las formas de neumotórax espontáneo debidas a la tuberculosis $(55,5 \%)$, sobre aquéllas debidas a infecciones agudas $(16 \%)$. Finalmente, al respecto, debemos hacer notar que el neumotórax espontáneo del niño menor siempre es secundario a tuberculosis de primo-infección, y aunque a primera visca parezca extrano, en el $80 \%$ de los casos se presenta en las formas no excavadas de tuberculosis y en sólo $20 \%$ en la forma de tuberculosis primaria excavada; este hecho se puede explicar por la presencia de lesiones bronquiales productoras del mecanismo valvular. En el niño mayor, en cambio, se presenta tanto en la tuberculosis primaria o postprimaria excavada $(80 \%)$ y sólo un minimo en las otras formas.

La forma de iniciación fué brusca en 9 casos e insidiosa en los restantes.

La forma de iniciación brusca fué aproximadamente de igual frecuencia en las formas secundarias a una enfermedad aguda (26.5\% de los casos) y en las formas secundarias a una tuberculosis $(22.2 \%)$.

Los síntomas que encontramos en estos 9 pacientes (iniciación brusca) se expresan en el cuadro No 6 .

Sin entrar en mayores comentarios sólo queremos señalar que en los casos de iniciación brusca de causa aguda, que en total suman 7 , en 4 de ellos $(57.1 \%$ ) existen antecedentes de un sarampiọn reciente (entre 1 y 4 meses antes) $y$ en 1 de una coqueluche (2 meses antes), dato que nos parece interesante si recordamos que en ambas afecciones son habituales las lesiones de peri-bronquitis y neumonitis intersticial y que no son raros los enfisemas pulmonares intersticiales y del mediastino. En todos 
Cuadro $N^{n} 6$

Iniciación y sintomas.

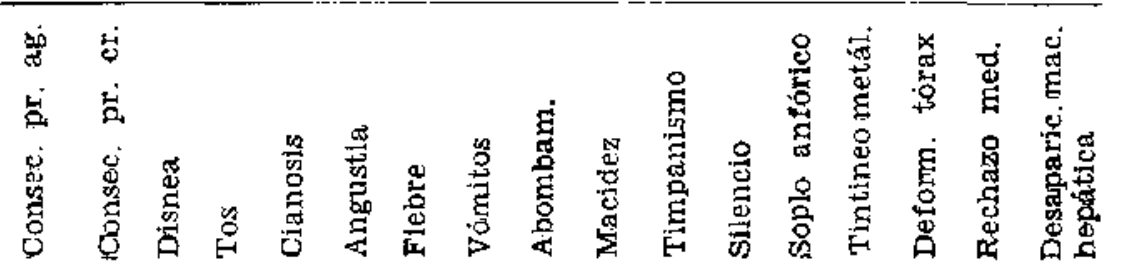

Injeiación

brusca

Inlelación

instidosa

9 casos

$7 \quad 2$

76

$7 \quad 9 \quad 10$

$\begin{array}{lllll}2 & 4 & 116 & 3 & 13\end{array}$

$\begin{array}{lllll}7 & 2 & 4 & 4\end{array}$

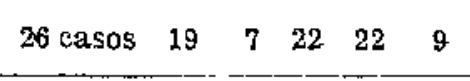

3

53

ellos se trataba de neumotórax a tensión.

La iniciación fué lenta e insidiosa, sin caracteres de agudeza y desprovistos de la dramaticidad de los casos anteriores en 26 enfermos. Sus síntomas predominantes fueron los que se describen en el cuadro No 6.

En estos enfermos no siempre el diagnóstico resultó fácil clínicamente, sea por la escasa intensidad de los síntomas clínicos, sea por tratarse de formas parciales. Para dar una idea aproximada de las dificultades, recordaremos que en sólo 10 casos se hizo el diagnóstico por sintomatología clínica; en 2 éste fué sospechado y en los 14 restantes fué el examen radiológico el que demostró su existencia. Los diagnósticos que se plantearon en estos casos fueron:

Derrame pleural: 5 .

Abscesos pulmonares: 5 .

Bronconeumonia: 1.

El neumotórax fué un hallazgo en un examen de rutina en otros 3 casos, que habían sufrido un proceso gripal poco antes. Por lo que respecta a la localización, 22 fueron derechos y 13 fueron izquierdos.

En 13 casos se trata de neumotórax total $y$ en 22 de neumotórax parcial de mayor o menor amplitud, con existencia de adherencias pleurales previas.

De ellos 12 fueron cerrados, 12 abiertos y 11 a tensión.

\section{Cuadro $N^{*} 7$}

$\begin{array}{ll}\text { Neumotórax totales } & 13 \\ \text { Neumotórax parciales } & 22 \\ \text { Neumotórax derechos } & 22 \\ \text { Neumotórax izquierdos } & 13 \\ \text { Neumotórax cerrados } & 12 \\ \text { Neumotórax abiertos } & 12 \\ \text { Neumotórax tensión } & 11\end{array}$

De las complicaciones debemos señalar la vómica, el hidroneumotórax y el pio-neumotorax, cuya presencia, según la etiología, es:

\section{Cuadro 8}

Complicaciones.

\begin{tabular}{lccc} 
& Vómica & $\begin{array}{c}\text { Hidro- } \\
\text { neumot. }\end{array}$ & $\begin{array}{c}\text { Plo- } \\
\text { neumot. }\end{array}$ \\
\hline $\begin{array}{c}\text { N. consecutiro } \\
\text { a causa aguda }\end{array}$ & 2 ceasos & 11 & 5 \\
$\begin{array}{c}\text { N. consecutívo } \\
\text { a causa crónica }\end{array}$ & 1 & 2 & 5
\end{tabular}

Es de notar que la vómica tuvo un efecto favorable en 2 niños que presentaban un pio-neumotórax consecutivo a una afección aguda, y en cambio, un efecto desfavorable (siembra) en un niño con tuberculosis.

Pronóstico y evolución: De los 35 casos fallecieron 15 en el Servicio (42.8\%) en las proporciones que indica el cuadro No 9 : 
Cuadro $\mathrm{N}^{\mathrm{9}} 9$

Fallecidos: 15

\begin{tabular}{lcccc}
\hline & -2 años -4 años +4 años & Duración \\
\hline N. consecutivos cuadros agudos & 4 & 1 & 3 & $\begin{array}{l}1 \text { a } 30 \text { dias } \\
\text { Media } 14.5 \text { dias }\end{array}$ \\
$\begin{array}{l}\text { N. consecutipos cuaćros crónicos: } \\
\text { Tuberculosis primaria }\end{array}$ & 2 & 2 & 1 & $\begin{array}{l}10 \text { horas a } 230 \text { dias } \\
\text { Media } 725 \text { dias } \\
6 \text { días a } 10 \text { días } \\
\text { Media } 8 \text { dias }\end{array}$ \\
\hline
\end{tabular}

Curados: 20

\begin{tabular}{|c|c|c|c|c|}
\hline & -2 años & -4 años & +4 años & Duración \\
\hline N. consecutivos cuadros agudos & 8 & 9 & 1 & $\begin{array}{l}14 \text { a } 300 \text { días } \\
\text { Medla } 75.5 \text { días }\end{array}$ \\
\hline \multicolumn{5}{|l|}{ N. consecutivos cuadros crónicos: } \\
\hline Tuberculosis primaria & o & 2 & 0 & 2 años \\
\hline Tuberculosis post primaria & 0 & $\mathbf{0}$ & 0 & \\
\hline
\end{tabular}

La observación del cuadro nos muestra algunos hechos, que aunque no permiten sacar conclusiones, nos parecen altamente sugerentes. Así, entre 22 neumotórax consecutivos a enfermedades agudas en menores de 4 años, fallecen solamente 5 , to que da una mortalidad, para este tipo y edad, de $22.7 \%$; de los niños mayores de 4 años fallecen 3 , lo que da un $75 \%$. Creemos que esta diferencia podria explicarse por la mayor facilidad con que se presenta este tipo de complicación en el niño menor, en quien no requiere, por lo tanto, seguir a afecciones generales graves $y$ destructoras. La mayor mortalidad del niño mayor es secundaria a afecciones generales graves y con gran compromiso local.

La sobrevida, después de producido el neumotórax y sus complicaciones, varió entre 1 y 21 días, con un promedio aritmético de 14.5 días. Creemos, por 1o tanto, que el pronóstico del neumotórax espontáneo depende, principalmente, del pronóstico de la enfermedad causal y de la repercusión sobre el estado general del niño.

En la tuberculosis vemos confirmado lo anterior, ya que en los menores de 4 años la mortalidad fué del $100 \%$; en un número reducido de casos la muerte se debió al neumotórax mismo y en la mayor parte se produjo por complicaciones de la afección tuberculosa (meningitis, diseminación).

En el niño mayor se trata, en todos los casos, de tuberculosis grave; la mortalidad alcanza un $60 \%$; de los fallecidos, en 2 se puede achacar directamente la muerte al neumotórax, que fué sofocante y a tensión; la terapéutica fracasó por la gran cantidad de adherencias y extensión de las lesiones. En los restantes la causa de muerte principal fué su afección tuberculosa.

De los niños mayores que sobreviven, 2 presentan un neumotórax crónico de 9 meses y 4 años de duración, aunque relativamente bien tolerados; se estudia la posibilidad de una intervención quirúrgica.

\section{Conclusiones.}

$1^{0}$ Se revisa la patogenia del neumotórax espontáneo.

$2^{\circ}$ De los 35 casos observados en el Hospital Roberto del Rio, el $74 \%$ corresponde a menores de 4 años y el resto a mayores de 4 años. 
$3^{\circ}$ Estos 35 casos corresponden a neumotórax espontáneos propiamente tales, libres de toda intervención pleural previa.

$4^{\circ}$ Veintiseis casos son secundarios a procesos pulmonares agudos, $\mathbf{9}$ a procesos pulmonares crónicos.

$5^{\circ}$ La iniciación brusca fué observada en la misma proporción en los casos agudos y en los crónicos (9 casos). La iniciación insidiosa (26 casos) también guardaba iguales proporciones.

$6^{\circ}$ La mortalidad en los niños menores de 4 años fué de $22.7 \%$ cuando el neumotórax espontáneo complicó cuadros agudos y de $100 \%$ cuando el neumotórax espontáneo fué secundario a una tuberculosis. En los niños mayores fué de $75 \%$ en los casos secundarios a cuadros agudos y de $60 \%$ cuando la causa desencadenante fué la tuberculosis.

\section{Summary.}

The authors review the pathogenia of spontaneous pneumothorax. Of the 35 cases studied at the Roberto del Río Hospital, $74 \%$ corresponded to children under 4 years of age. All these cases were true spontaneous pneumothorax with no previous history of pleural intervention. Twenty six cases were secondary to acute pulmonary processes and 9 to chronic pulmonary processes.

The acute onset was observed in the same proportion in the acute as in the chronic cases ( 9 cases). The insidious onset also kept the same proportion. Mortality in children under 4 years of age was $22,7 \%$ when spontaneous pneumotarax complicated an acute pulmonary process, reaching $100 \%$ when it was secondary to tuberculosis. In older children it was $75 \%$ in these cases secondary to acute pulmonary processes and $60 \%$ when it followed a tubercoulous process.

\section{Bibliografía.}

HASBUN, J , y MENTCGHELLO, J. - "Enfisema buloso en la infancia". Rev. Ch. de Ped. Vol. $19,1948$.

NEIRA, M. - "El neumotórax espontáneo en el lactante", Arch. Hosp. R. del Río 11: $115,1043$.
OYARCE, P. - Neumotórax espontáneo en el lactante". Areh. Hosp. Niños R. del Río 15: 88. 1947.

MATTE, R, y PULIDO, L. - "Neumotórax espontáneo". Rev. Ch. de Ped. 20: 198, 1049 .

ARIZTIA, A. - "Algunas consideraciones sobre neumotórax espontáneo y enfiseme en el niño menor". Rev. Ch. de Ped. $N^{\circ} 1$ : 1939.

TAPIA, M. - "Tuberculosis pulmonar". Tomo II, pág. 205.

PEILLIZA, J. M. - "Ploneumotórax espontáneo en la infancia". Buenos Aires.

CRENSHAW, G. L. - "Etiology, treatment and Surgical Indleations of non Tuberculous, non traumatic spontaneous Pneumothorax". Dis. of Chest. 17: 309, 1950.

CAST'EX, M. y MAZZEI. - "Neumotórax espontȧneo", Buenos Atres, 1941.

BREWER, DOLIEY and EVANS. - "The Surgical Management of Chronic Spontaneous Pneumothorax". Am. Ass. Thoracic Surgery. New orleans, 1949.

BERMARD, E. and MEYER, A. - "Diseases of the "Chest". "Treatment of non Tubeneulous spontaneous Pneumothorax. June 1951.

BROCK. - "Recurrent and Chronic spontaneous Pneumothorax". Thorax 1948, 388.

SAUERBRUCH. - "Cirugia del tórax".

SINGER, $G$ and BALLOU. - "Surgical diseases of the Chest". 1935.

MEYER, A.; NICO, J. P. and COTLENKO, N. - "Le Preumothorax spontané non Tuberculeux". Paris Medical, 1949, $10 \mathrm{Fe}$ vrier.

HANSEN, J. I. - "Spontaneoux Pneumothorax". Acta Médica Scandinávica 132: 517,1949 .

HOMKOWWR, G. M. - "Bilateral simultaneous Pneumothorax". British Med. Jour. 488: 1947.

VISLLLAC. - "Consideraciones sobre un caso de neumotórax espontáneo en el recién nacido". Rev. Soc. Pueric. 11: 159, 1945. Buenos Aires.

TEJEDA. - "Consideraciones sobre un caso de neumotórax espontáneo". Rev. Méd. Cubana 58 : 147,1947 .

SANTOS y SARMIENTO. - "Neumotórax espontáneo benigno crónico". Prensa Méd. Arg. 34: 1492: 1947.

HYLE, B. - "Benign idiopathic spontaneous Pneumothorax". Am. Journ. of the Miecical sciences 215: 427, 1948.

PARDAL y MAZZEr. - "Fl neumotórax espontáneo y la tuberculosis". Prensa Médica Argentina. 1935.

PARDAL y MAZZEI. - "El neumotórax espontáneo benigno, su patogenia, su diferenciación, su consideración como sindrome autónomo". Cátedra y Clinica 1: 87, 1934. 